\title{
Lineability of functionals and operators
}

\author{
by \\ Francisco Javier García-Pacheco (College Station, TX) and \\ DANiele Puglisi (Columbia, MO) \\ This paper is dedicated to the memory of Vladimir Gurariy
}

\begin{abstract}
This article is divided into two parts. The first one is on the linear structure of the set of norm-attaining functionals on a Banach space. We prove that every Banach space that admits an infinite-dimensional separable quotient can be equivalently renormed so that the set of norm-attaining functionals contains an infinite-dimensional vector subspace. This partially solves a question proposed by Aron and Gurariy. The second part is on the linear structure of dominated operators. We show that the set of dominated operators which are not absolutely summing is lineable.
\end{abstract}

1. Introduction to the lineability problem for functionals. We begin by introducing the following concepts related to the "algebraic size" of subsets of Banach spaces.

Definition 1.1 (Gurariy, 1991). A subset $M$ of a Banach space is said to be

(1) lineable if $M \cup\{0\}$ contains an infinite-dimensional vector subspace;

(2) dense-lineable if $M \cup\{0\}$ contains an infinite-dimensional dense vector subspace;

(3) spaceable if $M \cup\{0\}$ contains an infinite-dimensional closed vector subspace;

(4) $\mu$-lineable if $M \cup\{0\}$ contains a $\mu$-dimensional vector subspace, where $\mu$ is a cardinal number.

In order to have a better perspective of these new concepts, we refer the reader to the papers $\mathrm{G}$ ] and $\mathrm{AGS}$, where it is proved that several pathological properties occur more often than one might expect in connection with

2010 Mathematics Subject Classification: Primary 46B03; Secondary 47B38.

Key words and phrases: norm-attaining functional, separable quotient problem, biorthogonal system, dominated operator, absolutely summing operator. 
the definitions above. Framed in between lineability and geometry we found the following question.

Problem 1.2 (Aron and Gurariy, 2004). Given an infinite-dimensional Banach space $X$, is the set of norm-attaining functionals on $X, \operatorname{NA}(X)$, always lineable? Or, at least, can $X$ always be equivalently renormed to make $\mathrm{NA}(X)$ lineable?

We call this question the Lineability Problem for Functionals. In [BG] the following result related to this question was provided.

Theorem 1.3 (Bandyopadhyay and Godefroy, 2006). Let $X$ be an Asplund Banach space with the Dunford-Pettis property. The closed vector subspaces of $\mathrm{NA}(X)$ are finite-dimensional. In particular, $X$ cannot be equivalently renormed to make $\mathrm{NA}(X)$ spaceable.

In $[\mathrm{AG}$ the following theorem related to Problem 1.2 was proved.

Theorem 1.4 (Aizpuru and García-Pacheco, 2008). Let $X$ be an infinitedimensional Banach space. Fix $n \in \mathbb{N}$. Then $X$ can be equivalently renormed so that $\mathrm{NA}(X)$ is n-lineable.

In AAAG the following results related to Problem 1.2 were obtained.

Theorem 1.5 (Acosta, Aizpuru, Aron, and García-Pacheco, 2007). Let $K$ be an infinite compact Hausdorff topological space. Then $\mathrm{NA}(\mathcal{C}(K))$ is lineable.

Theorem 1.6 (Acosta, Aizpuru, Aron, and García-Pacheco, 2007). Let $X$ be an infinite-dimensional Banach space with a Schauder basis $\left(e_{n}, e_{n}^{*}\right)_{n \in \mathbb{N}}$ $\subset \mathrm{S}_{X} \times \mathrm{S}_{X^{*}}$. Then:

(1) If $\left(e_{n}\right)_{n \in \mathbb{N}}$ is monotone, then $\mathrm{NA}(X)$ is lineable.

(2) If $\left(e_{n}\right)_{n \in \mathbb{N}}$ is monotone and shrinking, then $\mathrm{NA}(X)$ is dense-lineable.

To finish this introduction on lineability of functionals we would like to present the following two questions proposed in [BG] that are closely related to Problem 1.2 .

Problem 1.7 (Bandyopadhyay and Godefroy, 2006).

(1) Does there exist a non-reflexive Banach space $X$ such that $\mathrm{NA}\left(X^{*}\right)$ is a vector space?

(2) Given an infinite-dimensional Banach space $X$, does $\mathrm{NA}(X)$ contain at least a 2-dimensional vector subspace?

As far as we know, these two questions still remain open.

2. Classical Banach spaces. In this section we will prove that the set of norm-attaining functionals is always lineable on spaces of continuous functions and always spaceable on spaces of integrable functions. 
THEOREM 2.1. Let $K$ be an infinite compact Hausdorff topological space. Let $X$ be a non-zero Banach space. Then $\operatorname{NA}(\mathcal{C}(K, X))$ is lineable.

Proof. We will show that every element in $\operatorname{span}\left\{x^{*} \circ \delta_{t}: t \in K\right\}$ is a norm-attaining functional, where $x^{*} \in \mathrm{S}_{X^{*}} \cap \mathrm{NA}(X)$. Let $t_{1}, \ldots, t_{p} \in K$ and $\lambda_{1}, \ldots, \lambda_{p} \in \mathbb{K} \backslash\{0\}$. Define the continuous function $f:\left\{t_{1}, \ldots, t_{p}\right\} \rightarrow[-1,1]$ as

$$
f\left(t_{i}\right)=\left|\lambda_{i}\right| / \lambda_{i} \quad \text { for } 1 \leq i \leq p .
$$

Since $K$ is normal and $\left\{t_{1}, \ldots, t_{p}\right\}$ is closed, by Urysohn's Lemma there exists a continuous extension $\tilde{f}: K \rightarrow[-1,1]$ of $f$. To finish, we note that $\|\tilde{f} x\|_{\infty}=1$, where $x \in \mathrm{S}_{X}$ and $x^{*}(x)=1$, and

$$
\left(\sum_{i=1}^{p} \lambda_{i}\left(x^{*} \circ \delta_{t_{i}}\right)\right)(\tilde{f} x)=\sum_{i=1}^{p}\left|\lambda_{i}\right|=\left\|\sum_{i=1}^{p} \lambda_{i}\left(x^{*} \circ \delta_{t_{i}}\right)\right\|_{\infty} .
$$

REMARK 2.2. Let $K$ be a compact Hausdorff topological space. Then $\mathcal{C}(K)$ has the Dunford-Pettis property (see, for instance, JL]). If, in addition, $K$ is scattered, then $\mathcal{C}(K)$ is Asplund (see [FHHMPZ] or [JL]). Hence, by Theorem 1.3. $\operatorname{NA}(\mathcal{C}(K))$ is not spaceable.

Lemma 2.3. Let $(\Omega, \Sigma, \mu)$ be a non-zero $\sigma$-finite measure space. Let $X$ be an Asplund Banach space. An element $f \in \mathrm{L}_{\infty}\left(\mu, X^{*}\right)$ attains its norm on $\mathrm{L}_{1}(\mu, X)$ if and only if there is a measurable set $A \in \Sigma$ with $\mu(A)>0$ such that, for all $t \in A, f(t)$ is norm-attaining and $\|f(t)\|=\|f\|_{\infty}$.

Proof. Suppose there is a measurable set $A \in \Sigma$ with $\mu(A)>0$ such that, for all $t \in A, f(t)$ is norm-attaining and $\|f(t)\|=\|f\|_{\infty}$. For every $t \in A$ there exists $g(t) \in \mathrm{S}_{X}$ such that $f(t)(g(t))=\|f(t)\|$. Since $(\Omega, \Sigma, \mu)$ is $\sigma$-finite, there is a measurable subset $B \subseteq A$ such that $0<\mu(B)<\infty$. Now,

$$
\frac{\chi_{B}}{\mu(B)} g \in \mathrm{S}_{\mathrm{L}_{1}(\mu, X)}
$$

and

$$
\int_{\Omega} f(t)\left(\frac{\chi_{B}}{\mu(B)} g(t)\right) d \mu(t)=\|f\|_{\infty} .
$$

Conversely, assume that $f \neq 0$ attains its norm at $g \in \mathrm{S}_{\mathrm{L}_{1}(\mu, X)}$. Then

$$
\|f\|_{\infty} \leq \int_{\Omega}|f(t)(g(t))| d \mu(t) \leq \int_{\Omega}\|f(t)\|\|g(t)\| d \mu(t) \leq\|f\|_{\infty}\|g\|_{1}=\|f\|_{\infty} .
$$

Take $C=\{t \in \Omega: g(t) \neq 0\}$. Clearly, $C$ is measurable and $\mu(C)>0$ since $g \neq 0$. Therefore, there exists a measurable set $Z_{1} \subset C$ such that $\mu\left(Z_{1}\right)=0$ and $|f(t)(g(t))|=\|f(t)\|\|g(t)\|$ for all $t \in C \backslash Z_{1}$. On the other hand, there exists a measurable set $Z_{2} \subset C$ such that $\mu\left(Z_{2}\right)=0$ and $\|f(t)\|=\|f\|_{\infty}$ for 
all $t \in C \backslash Z_{2}$. Finally, if $t \in A:=C \backslash\left(Z_{1} \cup Z_{2}\right)$, then $f(t)$ attains its norm at $g(t)$ and $\|f(t)\|=\|f\|_{\infty}$.

Theorem 2.4. Let $(\Omega, \Sigma, \mu)$ be a non-trivial $\sigma$-finite measure space. Let $X$ be a non-zero Asplund Banach space. Then $\mathrm{NA}\left(\mathrm{L}_{1}(\mu, X)\right)$ is spaceable.

Proof. By $(\Omega, \Sigma, \mu)$ being a non-trivial $\sigma$-finite measure space we mean that there exists a countably infinite family $\left(A_{n}\right)_{n \in \mathbb{N}}$ of pairwise disjoint measurable sets such that, for every $n \in \mathbb{N}, 0<\mu\left(A_{n}\right)<\infty$. Consider the infinite-dimensional closed subspace

$$
M:=\left\{\sum_{n=1}^{\infty} \alpha_{n} \chi_{A_{n}} x^{*}:\left(\alpha_{n}\right)_{n \in \mathbb{N}} \in c_{0}\right\}
$$

where $x^{*} \in \mathrm{NA}(X)$. Observe that $M$ is a closed subspace of $\mathrm{L}_{\infty}\left(\mu, X^{*}\right)$ linearly isometric to $c_{0}$. If $\left(\alpha_{n}\right)_{n \in \mathbb{N}} \in c_{0}$, then there exists $m \in \mathbb{N}$ such that $\left|\alpha_{m}\right|=\left\|\sum_{n=1}^{\infty} \alpha_{n} \chi_{A_{n}} x^{*}\right\|_{\infty}$. If $t \in A_{m}$, then $\left\|\sum_{n=1}^{\infty} \alpha_{n} \chi_{A_{n}} x^{*}(t)\right\|=\left|\alpha_{m}\right|$. From Lemma 2.3, we conclude that $M \subseteq \mathrm{NA}\left(\mathrm{L}_{1}(\mu, X)\right)$.

3. The separable case. In this section we will relate the Lineability Problem for Functionals to the Separable Quotient Problem (see [M] for a survey on the latter problem).

Lemma 3.1. Let $X$ be a Banach space. Assume that $M$ is a closed subspace of $X$. Then:

(1) If $M$ is 1-complemented and $\mathrm{NA}(M)$ is lineable, then $\mathrm{NA}(X)$ is lineable.

(2) If $M$ is proximinal and $\mathrm{NA}(X / M)$ is lineable, then $\mathrm{NA}(X)$ is lineable.

Proof. (1) It is sufficient to observe that if $p: X \rightarrow M$ is a linear projection of norm 1 , then $p^{*}(\mathrm{NA}(M)) \subseteq \mathrm{NA}(X)$.

(2) It is sufficient to notice that if $p: X \rightarrow X / M$ denotes the quotient map, then $p^{*}(\mathrm{NA}(X / M)) \subseteq \mathrm{NA}(X)$.

Observe that, taking into account Lemma 3.1, Theorem 2.1 is a direct consequence of Theorem 1.5 .

Lemma 3.2. Let $X$ be a Banach space. Assume that $M$ is a closed subspace of $X$. Then:

(1) If $M$ is complemented in $X$ and admits an equivalent norm for which $\mathrm{NA}(M)$ is lineable, then $X$ admits an equivalent norm for which $\mathrm{NA}(X)$ is lineable.

(2) If $X / M$ admits an equivalent norm for which $\mathrm{NA}(X / M)$ is lineable, then $X$ admits an equivalent norm for which $\mathrm{NA}(X)$ is lineable. 
Proof. (1) Let $N$ be a complement for $M$ in $X$. Assume that $M$ is already endowed with an equivalent norm for which NA(M) is lineable. Then $M \oplus_{2} N$ is isomorphic to $X$ and $\operatorname{NA}\left(M \oplus_{2} N\right)$ is lineable via Lemma 3.1(1).

(2) Let $|\cdot|$ be an equivalent norm on $X / M$ such that $\mathrm{NA}(X / M)$ is lineable. According to [BG, Lemma 2.4], there exists an equivalent norm $\|\cdot\|$ on $X$ which coincides with the original norm on $M$, whose quotient norm on $X / M$ is a positive multiple of $|\cdot|$, and which makes $M$ proximinal. To summarize, $X$ endowed with the norm $\|\cdot\|$ has a proximinal subspace $M$ such that $\mathrm{NA}(X / M)$ is lineable, and hence, by Lemma $3.1(2), \mathrm{NA}(X)$ is lineable.

Now, Theorem 1.6 together with the previous two lemmas will give us the following partial positive solution to Problem 1.2 .

Corollary 3.3. Let $X$ be a Banach space. If $X$ admits an infinitedimensional separable quotient, then $X$ can be equivalently renormed so that $\mathrm{NA}(X)$ is lineable.

Proof. According to [M], if $X$ admits an infinite-dimensional separable quotient, then $X$ admits an infinite-dimensional quotient $X / M$ with a Schauder basis. According to [D], $X / M$ can be endowed with an equivalent norm $|\cdot|$ so that $X / M$ has a monotone Schauder basis. In virtue of Theorem 1.6. $\mathrm{NA}(X / M)$ is lineable if $X / M$ is endowed with the equivalent norm $|\cdot|$. Finally, by Lemma $3.2(2), X$ admits an equivalent norm for which $\mathrm{NA}(X)$ is lineable.

4. The non-separable case. The next step is to obtain a similar version of Theorem 1.6 for non-separable spaces. We will then need an "uncountable" version of the Schauder basis concept: projection basis (see [JL, [P1], and the fourth chapter of [HMVZ] for a wider perspective).

Definition 4.1 (Banach, 1932; Markushevich, 1943; Plichko, 1983). Let $X$ be a Banach space. Let $\left(x_{i}, x_{i}^{*}\right)_{i \in I} \subseteq X \times X^{*}$. We say that the system $\left(x_{i}, x_{i}^{*}\right)_{i \in I}$ is

(1) biorthogonal if $x_{i}^{*}\left(x_{j}\right)=\delta_{i, j}$ for all $i, j \in I$;

(2) fundamental if $\operatorname{span}\left\{x_{i}: i \in I\right\}=X$;

(3) total if $\bigcap_{i \in I} \operatorname{ker}\left(x_{i}^{*}\right)=\{0\}$;

(4) a Markushevich basis if it is biorthogonal, fundamental, and total;

(5) a projection basis if it is a Markushevich basis and $I$ can be wellordered in such a way that for all $i \in I$ there exist collectively bounded projections $P_{i}: X \rightarrow \overline{\operatorname{span}}\left\{x_{j}: j<i\right\}$ parallel to $\overline{\operatorname{span}}\left\{x_{j}:\right.$ $j \geq i\}$.

(6) a monotonic projection basis if it is a projection basis where the norms of all the projections are equal to 1. 
The following is a very helpful characterization of Banach spaces admitting a fundamental and biorthogonal system in terms of quotients and monotonic projection bases (see [P2]).

Theorem 4.2 (Plichko, 1983). Let $X$ be a non-separable Banach space. The following conditions are equivalent:

(1) $X$ admits a fundamental and biorthogonal system.

(2) $X$ has a quotient that admits a monotonic projection basis of cardinality dens $(X)$, the density character of $X$.

Unfortunately, there are non-separable Banach spaces that do not admit fundamental and biorthogonal systems (see [GK] and [P2]).

Remark 4.3 (Godun and Kadets, 1980; Plichko, 1983). For an indexset $\Gamma$ of cardinality greater than $2^{\aleph_{0}}$ the space $\ell_{\infty}^{c}(\Gamma)$ (the closed subspace of $\ell_{\infty}(\Gamma)$ consisting of vectors with countable support) does not admit a fundamental and biorthogonal system.

The non-separable version of Theorem 1.6 follows.

TheOREM 4.4. Let $X$ be a Banach space that admits a monotonic projection basis $\left(x_{i}, x_{i}^{*}\right)_{i \in I} \subseteq X \times X^{*}$. Then $\mathrm{NA}(X)$ is card $(I)$-lineable.

Proof. Consider the infinite-dimensional subspace of $X^{*}$ given by $M:=$ $\operatorname{span}\left\{x_{i}^{*}: i \in I\right\}$. Let $x^{*}=\lambda_{1} x_{i_{1}}^{*}+\cdots+\lambda_{n} x_{i_{n}}^{*} \in M$. Since $\left(x_{i}, x_{i}^{*}\right)_{i \in I}$ is fundamental, biorthogonal, and monotonic, we have

$$
\sup x^{*}\left(\mathrm{~B}_{X}\right)=\sup x^{*}\left(\mathrm{~B}_{X} \cap \operatorname{span}\left\{x_{i_{1}}, \ldots, x_{i_{n}}\right\}\right),
$$

and the above sup is attained since the set

$$
\mathrm{B}_{X} \cap \operatorname{span}\left\{x_{i_{1}}, \ldots, x_{i_{n}}\right\}=\mathrm{B}_{\mathrm{span}\left\{x_{i_{1}}, \ldots, x_{i_{n}}\right\}}
$$

is compact. Therefore, each element of $M$ attains its norm, and hence $\mathrm{NA}(X)$ is $\operatorname{card}(I)$-lineable.

As a corollary, we obtain a result that could be seen as a non-separable version of Corollary 3.3 .

Corollary 4.5. Let $X$ be a non-separable Banach space that admits a fundamental biorthogonal system. Then $X$ can be equivalently renormed so that $\operatorname{NA}(X)$ is dens $(X)$-lineable.

Proof. By Theorem 4.2, $X$ admits a quotient $X / M$ with a monotonic projection basis of cardinality dens $(X)$. Then $\mathrm{NA}(X / M)$ is dens $(X)$-lineable in view of Lemma 4.4. Finally, by Lemma 3.2 (2), $X$ admits an equivalent norm for which $\mathrm{NA}(X)$ is dens $(X)$-lineable. 
5. Preliminaries on dominated operators. Our results on dominated operators are a continuation of the study of lineability of sets of operators initiated in [PS]. Following [DJT] we recall the following definitions.

Definition 5.1 (Grothendieck, 1955). Let $X$ and $Y$ be Banach spaces. A bounded linear operator $T \in \mathcal{L}(X, Y)$ is said to be absolutely summing if for each unconditionally convergent series $\sum_{i=1}^{\infty} x_{i}$ in $X$, the series $\sum_{i=1}^{\infty} T\left(x_{i}\right)$ is absolutely convergent.

Absolutely summing operators can be characterized as follows.

TheOrem 5.2 (Grothendieck, 1955). Let $X$ and $Y$ be Banach spaces. A bounded linear operator $T \in \mathcal{L}(X, Y)$ is absolutely summing if and only if there exists a constant $C \geq 0$ such that for any natural number $m$ and any choice of $x_{1}, \ldots, x_{m}$ in $X$ we have

$$
\sum_{i=1}^{m}\left\|T\left(x_{i}\right)\right\| \leq C \sup \left\{\sum_{i=1}^{m}\left\langle x^{*}, x_{i}\right\rangle x^{*} \in \mathrm{B}_{X^{*}}\right\} .
$$

The space $\Pi_{1}(X, Y)$ of absolutely summing operators from $X$ to $Y$ becomes a Banach space when endowed with the norm $\pi_{1}$, which is the least constant $C$ for which the inequality above holds.

Definition 5.3 (Pietsch, 1965). Let $X$ and $Y$ be Banach spaces. Let $K$ be a Hausdorff compact topological space. A bounded linear operator $T: \mathcal{C}(K, X) \rightarrow Y$ is said to be dominated if there exists a positive regular measure $\nu$ on $\mathcal{B}(K)$ such that

$$
\|T(f)\| \leq \int_{K}\|f(t)\| d \nu(t) \quad \text { for all } f \in \mathcal{C}(K, X) .
$$

In [PI, 2.3.4], Pietsch showed the following result.

TheOREM 5.4 (Pietsch, 1965). Let $K$ be a compact Hausdorff topological space. Let $X$ and $Y$ be Banach spaces, and $T: \mathcal{C}(K, X) \rightarrow Y$ a bounded linear operator. Then:

(1) If $T$ is absolutely summing, then $T$ is dominated.

(2) If $X$ is finite-dimensional, then $T$ is dominated if and only if $T$ is absolutely summing.

From [PS] we know that dominated operators on spaces of continuous functions with values in infinite-dimensional Banach spaces are actually far from being absolutely summing. Finally, in the next section we will need the following fundamental and spectacular result in analysis (see [DR] and also [DJT, 1.2]).

Theorem 5.5 (Dvoretzky-Rogers, 1950). Let X be a Banach space. The following are equivalent: 
(1) $X$ is finite-dimensional.

(2) Every unconditionally convergent series in $X$ is absolutely convergent.

6. Dominated operators which are not absolutely summing. In this section we will prove that the set of dominated operators that are not absolutely summing is lineable. We will need the following lemma.

Lemma 6.1. Let $X$ be a Banach space and $K$ a compact Hausdorff topological space. Let $\sum_{k=1}^{\infty} x_{k}$ be an unconditionally convergent series in $X$ and let $\left(\phi_{k}\right)_{k \in \mathbb{N}} \subseteq \mathcal{C}(K)$ be such that $\left\|\phi_{k}\right\|_{\infty} \leq 1$ for all $k \in \mathbb{N}$. Then $\sum_{k=1}^{\infty} \phi_{k} x_{k}$ is unconditionally convergent in $\mathcal{C}(K, X)$.

Proof. Let $\sigma: \mathbb{N} \rightarrow \mathbb{N}$ be a permutation. It suffices to note that $\left(\phi_{k}(t)\right)_{k \in \mathbb{N}}$ $\in \mathrm{B}_{\ell_{\infty}}$ for each $t \in K$. By [DJT, 1.9] we have

$$
\left\|\sum_{k \in \mathbb{N}} \phi_{\sigma(k)} x_{\sigma(k)}\right\|=\sup _{t \in K}\left\|\sum_{k \in \mathbb{N}} \phi_{\sigma(k)}(t) x_{\sigma(k)}\right\| \leq\left\|\sum_{k \in \mathbb{N}} x_{k}\right\|<\infty .
$$

Finally, we are in a position to state and prove the main result in this section.

TheOrem 6.2. Let $X$ be an infinite-dimensional Banach space and $K$ an infinite compact Hausdorff topological space. The set of dominated operators from $\mathcal{C}(K, X)$ to $X$ that are not absolutely summing is lineable. If, in addition, $K$ is sequentially compact and $X$ contains $\ell_{1}$ isometrically, then the set of dominated operators from $\mathcal{C}(K, X)$ to $X$ that are not absolutely summing is $\ell_{1}$-spaceable.

Proof. Since $X$ is infinite-dimensional, according to the Dvoretzky-Rogers Theorem there exists a sequence $\left(x_{k}\right)_{k \in \mathbb{N}}$ in $X$ such that $\sum_{k=1}^{\infty} x_{k}$ is unconditionally convergent but not absolutely convergent. Pick a sequence $\left(t_{n}\right)_{n \in \mathbb{N}}$ of points of $K$ such that $t_{i} \neq t_{j}$ if $i \neq j$. For every $n \in \mathbb{N}$, define

$$
T_{n}: \mathcal{C}(K, X) \rightarrow X, \quad \phi \mapsto T_{n}(\phi):=\phi\left(t_{n}\right) .
$$

We will consider the vector space $\operatorname{span}\left\{T_{n}: n \in \mathbb{N}\right\}$. So, fix $m \in \mathbb{N}$ and $c_{1}, \ldots, c_{m} \in \mathbb{K}$ not all zero:

(1) If $c_{1} T_{1}+\cdots+c_{m} T_{m}=0$, then $c_{1}=\cdots=c_{m}=0$ : Indeed, for every $i \in\{1, \ldots, m\}$ define

$$
\psi_{i}:\left\{t_{1}, \ldots, t_{m}\right\} \rightarrow\{0,1\}, \quad t_{j} \mapsto \psi_{i}\left(t_{j}\right)=\delta_{i j} .
$$

Since $\left\{t_{1}, \ldots, t_{m}\right\}$ is closed in $K$ and $K$ is normal, we may extend $\psi_{i}$ to a continuous function on $K$ that we will keep denoting by $\psi_{i}$. Now pick any $x \in X \backslash\{0\}$ and evaluate

$$
0=\left(c_{1} T_{1}+\cdots+c_{m} T_{m}\right)\left(\psi_{i} x\right)=c_{i} x .
$$


(2) $c_{1} T_{1}+\cdots+c_{m} T_{m}$ is not absolutely summing: Indeed, there exists $i \in\{1, \ldots, m\}$ such that $c_{i} \neq 0$. According to Lemma 6.1, $\sum_{k=1}^{\infty} \psi_{i} x_{k}$ is unconditionally convergent in $\mathcal{C}(K, X)$. Then

$$
\left(c_{1} T_{1}+\cdots+c_{m} T_{m}\right)\left(\sum_{k=1}^{\infty} \psi_{i} x_{k}\right)=c_{i} \sum_{k=1}^{\infty} x_{k} .
$$

(3) $c_{1} T_{1}+\cdots+c_{m} T_{m}$ is a dominated operator: Indeed, for every $\phi \in$ $\mathcal{C}(K, X)$,

$$
\left(c_{1} T_{1}+\cdots+c_{m} T_{m}\right)(\phi)=\int_{K} \phi d\left(c_{1} \delta_{t_{1}}+\cdots+c_{m} \delta_{t_{m}}\right),
$$

where $\delta_{x}$ denotes the Dirac measure at $x$.

We now assume that $K$ is sequentially compact and $X$ contains $\ell_{1}$ isometrically. We can then assume that $t_{n} \rightarrow t_{\infty} \in K \backslash\left\{t_{n}: n \in \mathbb{N}\right\}$. Bearing in mind that $K$ is Hausdorff, for every $n \in \mathbb{N}$ we can find an open neighborhood $U_{n}$ of $t_{n}$ such that $U_{i} \cap U_{j}=\emptyset$ if $i \neq j$. Fix $n \in \mathbb{N}$. We can define the following continuous function:

$$
\varphi_{n}:\left\{t_{n}\right\} \cup\left(K \backslash U_{n}\right) \rightarrow[0,1], \quad t \mapsto \varphi_{n}(t)= \begin{cases}1 & \text { if } t=t_{n} \\ 0 & \text { otherwise }\end{cases}
$$

Since $\left\{t_{n}\right\} \cup\left(K \backslash U_{n}\right)$ is closed and $K$ is normal, we can extend $\varphi_{n}$ to a $[0,1]$-valued function defined on the whole of $K$ which we will keep denoting by $\varphi_{n}$. The sequence $\left(\varphi_{n}\right)_{n \in \mathbb{N}}$ enjoys the following property: if $t \in K$, then $\sum_{n=1}^{\infty} \varphi_{n}(t) \leq 1$. Indeed, if $\sum_{n=1}^{\infty} \varphi_{n}(t)>1$, then there are least two indices, say $i \neq j$, for which $\varphi_{i}(t)$ and $\varphi_{j}(t)$ are strictly positive. This means that $t \in U_{i} \cap U_{j}$, which is impossible. We will consider the closed vector space $\left\{\sum_{n=1}^{\infty} \lambda_{n} T_{n}:\left(\lambda_{n}\right)_{n \in \mathbb{N}} \in \ell_{1}\right\}$. So, fix $\left(\lambda_{n}\right)_{n \in \mathbb{N}} \in \ell_{1} \backslash\{0\}$ :

(1) $\left\|\sum_{n=1}^{\infty} \lambda_{n} T_{n}\right\|=\sum_{n=1}^{\infty}\left|\lambda_{n}\right|$ : Indeed, let $\left(e_{n}\right)_{n \in \mathbb{N}}$ denote the canonical basis of $\ell_{1}$. For all $t \in K,\left\|\sum_{n=1}^{\infty} \varphi_{n}(t) e_{n}\right\|=\sum_{n=1}^{\infty} \varphi_{n}(t) \leq 1$. Therefore, $\left\|\sum_{n=1}^{\infty} \varphi_{n} e_{n}\right\| \leq 1$. Finally,

$$
\left(\sum_{n=1}^{\infty} \lambda_{n} T_{n}\right)\left(\sum_{m=1}^{\infty} \varphi_{m} e_{m}\right)=\sum_{n=1}^{\infty} \lambda_{n} e_{n},
$$

which indicates that

$$
\left\|\sum_{n=1}^{\infty} \lambda_{n} T_{n}\right\|=\sum_{n=1}^{\infty}\left|\lambda_{n}\right| .
$$

(2) $\sum_{n=1}^{\infty} \lambda_{n} T_{n}$ is not absolutely summing: Indeed, there exists $i \in \mathbb{N}$ such that $\lambda_{i} \neq 0$. According to Lemma 6.1. $\sum_{k=1}^{\infty} \varphi_{i} x_{k}$ is uncondi- 
tionally convergent in $\mathcal{C}(K, X)$. Then

$$
\left(\sum_{n=1}^{\infty} \lambda_{n} T_{n}\right)\left(\sum_{k=1}^{\infty} \varphi_{i} x_{k}\right)=\lambda_{i} \sum_{k=1}^{\infty} x_{k} .
$$

(3) $\sum_{n=1}^{\infty} \lambda_{n} T_{n}$ is a dominated operator: Indeed, for every $\phi \in \mathcal{C}(K, X)$,

$$
\left(\sum_{n=1}^{\infty} \lambda_{n} T_{n}\right)(\phi)=\int_{K} \phi d\left(\sum_{n=1}^{\infty} \lambda_{n} \delta_{t_{n}}\right)
$$

Acknowledgements. The first author would like to thank Prof. Aron for his valuable encouragement. This paper was written while the second author was visiting the Department of Mathematics at Texas A\&M University. He would like to thank the first author for his nice and warm hospitality. The second author was supported by NSF Grant DMS-0652684 (FRG: Fourier Analytic and Probabilistic Method in Geometric Functional Analysis and Convexity).

\section{References}

[AAAG] M. D. Acosta, A. Aizpuru, R. M. Aron, and F. J. García-Pacheco, Functionals that do not attain their norm, Bull. Belg. Math. Soc. Simon Stevin 14 (2007), 407-418.

[AG] A. Aizpuru and F. J. García-Pacheco, A note on $\mathrm{L}^{2}$-summand vectors in dual Banach spaces, Glasgow J. Math. 50 (2008), 429-432.

[AGS] R. M. Aron, V. I. Gurariy and J. B. Seoane-Sepúlveda, Lineability and spaceability of sets of functions on $\mathbb{R}$, Proc. Amer. Math. Soc. 133 (2005), 795-803.

[BG] P. Bandyopadhyay and G. Godefroy, Linear structure in the set of normattaining functionals on a Banach space, J. Convex Anal. 13 (2006), 489497.

[D] J. Diestel, Sequences and Series in Banach Spaces, Grad. Texts in Math. 92, Springer, New York, 1984.

[DJT] J. Diestel, H. Jarchow, and A. Tonge, Absolutely Summing Operators, Cambridge Univ. Press, 1995.

[DR] A. Dvoretzky and C. A. Rogers, Absolute and unconditional convergence in normed linear spaces, Proc. Nat. Acad. Sci. U.S.A. 36 (1950), 192-197.

[FHHMPZ] M. Fabian, P. Habala, P. Hájek, V. Montesinos-Santalucía, J. Pelant, and V. Zizler, Functional Analysis and Infinite-Dimensional Geometry, CMS Books Math. 8, Springer, 2001.

[HMVZ] P. Hájek, V. Montesinos, J. Vanderwerff, and V. Zizler, Biorthogonal Systems in Banach Spaces, CMS Books Math. 26, Springer, 2008.

[GK] B. V. Godun and M. Kadets, Banach spaces without complete minimal systems, Funktsional. Anal. i Prilozhen. 14 (1980), no. 4, 67-68 (in Russian).

[G] V. I. Gurariy, Linear spaces composed of everywhere nondifferentiable functions, C. R. Acad. Bulgare Sci. 44 (1991), 13-16.

[JL] W. Johnson and J. Lindenstrauss (eds.), Handbook of the Geometry of Banach Spaces, North-Holland, Amsterdam, 2003. 
[M] J. Mujica, Separable quotients of Banach spaces, Rev. Mat. Univ. Complut. Madrid 10 (1998), 299-330.

[PI] A. Pietsch, Nukleare lokalkonvexe Räume, Akademie-Verlag, Berlin, 1965.

[P1] A. N. Plichko, Banach spaces without fundamental biorthogonal systems, Soviet Math. Dokl. 254 (1980), 978-801.

[P2] - Fundamental biorthogonal systems and projection bases in Banach spaces, Mat. Zametki 33 (1983), 473-476 (in Russian).

[PS] D. Puglisi and J. B. Seoane-Sepúlveda, Bounded linear non-absolutely summing operators, J. Math. Anal. Appl. 338 (2008), 292-298.

Francisco Javier García-Pacheco

Department of Mathematical Sciences

Texas A\&M University

College Station, TX 77843-3368, U.S.A.

E-mail: fgarcia@math.tamu.edu
Daniele Puglisi

Department of Mathematics

University of Missouri

Columbia, MO 65201, U.S.A.

E-mail: puglisi@math.missouri.edu

Received January 27, 2010

Revised version May 23, 2010 